\title{
Avances y límites de la sustentabilidad social
}

\author{
GuILlermo Foladori*
}

\begin{abstract}
When speaking on the environment it is common to consider only the external nature. However, all the discussion on the modern environmental crisis, and a more healthful environment for human development consideres human society as a part of the environment. The concept of sustainable development was born including not only ecological, but also social and economical sustainability. In the paper it is intended to analyze the way social sustainability has been used in the sustainable development discourse. It is concluded that although there has been an advance on social sustainability in development policies as well as in academic discussion, there are certain limits to its feasibility which are imposed by the capitalist system.
\end{abstract}

Keywords: social sustainability, sustainable development, social participation, environmental crisis.

\section{Resumen}

Cuando se habla de medio ambiente en términos comunes, se está considerando la naturaleza externa al ser humano. No obstante, toda la discusión sobre la moderna crisis ambiental y sobre una alternativa ambientalmente más saludable para el desarrollo humano considera a la sociedad humana como parte del medio ambiente. El propio concepto de desarrollo sustentable desde su nacimiento incorporó una sustentabilidad social y económica a la sustentabilidad ambiental.

En este artículo se analiza ese camino de la sustentabilidad social o socio-ambiental en el discurso del desarrollo sustentable. Llegamos a la conclusión de que, aun cuando se haya avanzado en el tema de la sustentabilidad social, tanto en las políticas de desarrollo como en la discusión académica existen ciertas barreras colocadas por la propia lógica de funcionamiento del sistema capitalista, que limitan la viabilidad de la sustentabilidad social.

Palabras clave: sustentabilidad social, desarrollo sustentable, crisis ambiental, participación social.

*Columbia University. Correo-e: fola@ufpr.br 


\section{La tridimensionalidad de la sustentabilidad}

La conciencia de la moderna crisis ambiental cristalizó a finales de la década de los sesenta y comienzos de los setenta con una serie de libros, congresos y encuentros internacionales que mostraron, todos ellos, la necesidad de rediscutir el desarrollo debido a los estragos que él mismo estaba generando en la naturaleza externa. No obstante, y a pesar del amplio abanico de posiciones, la preocupación por la naturaleza externa reflejaba intereses humanos, una vez que los niveles de contaminación ambiental o de depredación de los recursos naturales parecían colocar en jaque las posibilidades del capitalismo de continuar su crecimiento ilimitado. De manera que la preocupación por el desarrollo humano estaba por detrás y conducía las preocupaciones por la naturaleza externa.

Una vez que surge el concepto de desarrollo sustentable y adquiere fuerza cuando es divulgado por el informe Nuestro Futuro Común (WCED, 1987), desaparecieron las pocas dudas que aún existían acerca de si la preocupación por la naturaleza debía o no considerar al ser humano. El desarrollo sustentable incorpora a la conservación de la naturaleza externa (sustentabilidad ecológica), la sustentabilidad social, y también una sustentabilidad económica. ${ }^{1}$ Sin embargo algunos autores, instituciones y prácticas de política ambiental continúan privilegiando o considerando exclusivamente a la sustentabilidad ambiental. ${ }^{2}$ A pesar de existir decenas, $o$ tal vez cientos de definiciones sobre desarrollo sustentable, una vez que dichas definiciones son analizadas y explicadas, en la mayoría de los casos los aspectos sociales y económicos de la sustentabilidad siempre complementan los de la sustentabilidad ecológica. El esquema I, elaborado por el World Resources Institute (WRI, 2002) es un ejemplo visual contundente de esa tridimensionalidad de la sustentabilidad (véase el esquema I). El ángulo superior representa a la sustentabilidad económica, el inferior derecho a la ecológica y el inferior izquierdo a la social.

\footnotetext{
${ }^{1}$ Algunos autores agregan otras 'sustentabilidades', como la política, cultural, etcétera; estas últimas, no obstante, pueden ser incluidas en la sustentabilidad social. El concepto anterior y algo diferente de ecodesarrollo pregonaba cinco aspectos: social, económico, espacial y cultural (Sachs, 1994).

${ }^{2}$ Según Caldwell y Hon (1984) el concepto de desarrollo ecológicamente sustentable estuvo presente implícitamente en la Conferencia Intergubernamental de Expertos en las Bases Científicas para el Uso Racional y la Conservación de la Biosfera (Biosphere Conference) realizada en París, en septiembre de 1968, así como en la Conferencia sobre Aspectos Ecológicos del Desarrollo Internacional, realizada en diciembre de 1968 en las cercanías de Washington y organizada por The Conservation Foundation y el Center for the Biology of Natural Systems de la Universidad de Washington.
} 


\section{Esquema 1 \\ Three arenas for assessing performance}

\section{Three Arenas for Assessing Performance}

Key Concept: The Triple Bottom Line

Sustainability Simplified

Growth

Shareholder value

Efficiency

Empowerment \& Equity

Social mobility

Social cohesion

Cultural identity

Institutional development

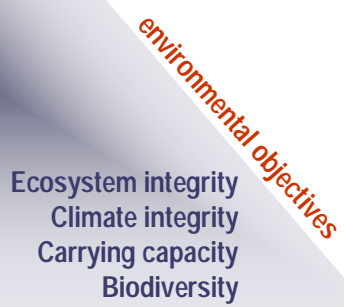

wante resouters inctituse

Fuente: WRI, 2002.

Esta presentación de la tridimensionalidad de la sustentabilidad resulta atractiva y parece abarcar a los diferentes sectores para los cuales el desarrollo capitalista debe prestar atención. No obstante, y como pretendemos mostrar en el siguiente apartado, se trata de una visión tecnicista y, por tanto, ideológicamente comprometida con el propio capitalismo causante de tanta degradación.

\section{El carácter técnico de la tridimensionalidad}

Al transitar de los esquemas visuales o teóricos a las prácticas políticas derivadas de ellos, se comprende realmente el significado de una sustentabilidad económica, social o ecológica. En efecto, aunque difícil de medir, la sustentabilidad ecológica es la que resulta de menores desacuerdos: la sustentabilidad ecológica se refiere a un cierto equilibrio y mantenimiento de los ecosistemas, la conservación y el mantenimiento de un caudal genético de las especies, que garantice la resiliencia frente a los impactos externos. A pesar de que en el esquema 1 no es explícita, la sustentabi- 
lidad ecológica incluye también el mantenimiento de los recursos naturales abióticos y lo que se denomina integridad climática, que no es otra cosa que un clima con la menor interferencia humana posible. En su conjunto, la sustentabilidad ecológica corresponde al concepto de conservación de la naturaleza, en el sentido de naturaleza externa al ser humano. El criterio para 'medir' la sustentabilidad ecológica, aunque eso sea técnicamente imposible, está dado por el concepto de integridad, o naturaleza sin modificaciones hechas por el ser humano (Pimentel et al., 2000). Así, cuanto más cerca se está de la naturaleza 'intocada' o 'prístina', más ecológicamente sustentable es; cuanto más humanamente modificada esté la naturaleza, menor sustentabilidad ecológica habrá. Al final, a pesar de las diferentes posiciones, la 'medida' es siempre la naturaleza no humana y, por lo tanto, la posición de los 'preservacionistas' que defienden la menor transformación de la naturaleza se constituye en la brújula que orienta.

El concepto de sustentabilidad económica comienza a complicar el análisis de la sustentabilidad. Si ese concepto se restringe al crecimiento económico y a la eficiencia productiva, surge la pregunta de si un crecimiento ilimitado -como aquel comandado por el sistema capitalista- es congruente con la sustentabilidad ambiental (Rees y Wackernagel, 1999). Para los representantes de la más ortodoxa economía ecológica, el crecimiento no puede ser ilimitado; de manera que, para ser viable, el capitalismo debería convertirse en un proyecto de cero crecimiento (Daly, 1989). No obstante, como el crecimiento ilimitado es intrínseco a la dinámica capitalista, dicha tesis sería equivalente a negar el capitalismo sin tener nada que colocar en su lugar. Para las vertientes más blandas de la economía ecológica, y para los economistas ambientales, bastaría corregir los procesos productivos para obtener un desarrollo capitalista sustentable (Pearce y Turner, 1995). Básicamente, se trataría de sustituir crecientemente los recursos naturales no renovables por los renovables, y también de disminuir tendencialmente la contaminación.

Por último, el concepto de sustentabilidad social es, tal vez, el que ha generado mayores polémicas y el que más ha cambiado en su contenido durante los últimos treinta años. Un estudioso de la evolución del concepto de desarrollo sustentable señala la diferencia entre sustentabilidad social y ecológica como un gran problema conceptual:

Differentiating between ecological and social sustainability could be a first step toward clarifying some of the discussion (Lélé, 1991: 615). 
Hasta la década de los noventa, dos temáticas concentraban la discusión en torno a la sustentabilidad social: la pobreza y el incremento poblacional. No obstante, no era simple distinguir la cuestión social de la ecológica. El propio Lélé anotaba que la erosión del suelo podía ser considerado un problema de insustentabilidad ecológica, pero si aquélla fuese causada por cultivar en tierras marginales y por comunidades pobres sin recursos sería simultáneamente un problema de insustentabilidad social (Lélé, 1991: 610).

En un artículo publicado en el año 2000 (Foladori y Tommasino, 2000) argumentábamos que hasta la década del noventa el concepto de sustentabilidad social no era utilizado con fines auténticos; por el contrario, su utilización tenía el espurio fin de encubrir el interés por la sustentabilidad ecológica. Puesto de otra forma: para las instituciones internacionales como la onU o el Banco Mundial entre otras, la pobreza y/o el incremento poblacional no eran considerados como un problema de insustentabilidad en sí mismo, sino en la medida en que causaran insustentabilidad ecológica. Dos ejemplos facilitarán su entendimiento. El primero puede ser aquel mostrado por Lélé, de campesinos pobres que por la falta de recursos degradan el suelo provocando erosión. El problema de insustentabilidad en dicho caso no es la pobreza per se, sino el resultado de la erosión del suelo. Es decir que aun cuando se considere a la pobreza como un problema de insustentabilidad social, lo que verdaderamente interesa es la (in)sustentabilidad ecológica que provoca.

El segundo ejemplo es el del crecimiento poblacional. Los pobres se reproducen más que otros estratos de la población. En este caso, el problema de insustentabilidad social es el crecimiento de la pobreza. Sin embargo, el interés explícito -aunque no evidente- es que el aumento de la población presiona sobre los recursos e incrementa los residuos. Tanto en el primero como en el segundo ejemplos, la insustentabilidad social es utilizada en la medida en que se constituya en elemento que afecte la sustentabilidad ecológica. Se trata, evidentemente, de una sustentabilidad social limitada. En aquel artículo llamábamos a esa forma de entender la sustentabilidad social como "puente, en la medida en que el interés por la sustentabilidad social era simplemente el de alcanzar una meta ecológica para la cual la sustentabilidad social se constituía en un instrumento o medio" (Foladori y Tommasino, 2000). En el mismo año 2000, Anand y Sen llegaban a la misma conclusión, y criticaban al Banco Mundial, después de 
una cita de un informe de esa institución con las siguientes palabras: ${ }^{3}$

this argument provides an instrumental justification for poverty alleviation, as a means of protecting the environment (Anand y Sen, 2000: 2038).

Ellos también encontraron que la forma en que el Banco Mundial considera la sustentabilidad social era solamente un medio para alcanzar la ecológica. Es necesario, entonces, no confundir las palabras pobreza, migraciones, hambre, etcétera, con sustentabilidad social, ya que, en muchos casos esas palabras son utilizadas para esconder el verdadero interés que son los recursos naturales. Muchas veces, las capacidades humanas y las relaciones sociales, que se refieren a la forma en que se genera la pobreza, o el desempleo, no están en discusión, sino solamente sus consecuencias técnicas en tanto contaminación y degradación de los ecosistemas. Esto ha sido denunciado en varios discursos hasta por un ex vice presidente del Banco Mundial. En febrero de 1999, el economista Joseph Stiglitz hablaba sobre el papel de la participación social como un fin en sí mismo, además de un medio para incrementar el crecimiento económico:

The central argument of this paper has been that open, transparent, and participatory processes are important ingredients in the development transformation -important both for sustainable economic development and for social development that should be viewed as an end in itself and as a means to more rapid economic growth (Stiglitz, 1999: 9).

Más allá de las voces de denuncia de la sustentabilidad social como medio y no como fin, lo que tienen en común esas formas de considerar la sustentabilidad, sea la ecológica o la social, es su perspectiva técnica. Esto es de extrema importancia, porque se refiere al hecho de reducir la polémica sobre desarrollo sustentable a los cambios dentro del sistema capitalista.

$\mathrm{Al}$ operar cualquier transformación de la naturaleza, el ser humano establece dos tipos de relaciones, que pueden distinguirse tanto desde el punto de vista técnico como práctico. Los seres humanos establecen relaciones técnicas con el medio ambiente externo. Esas relaciones técnicas son las que permiten que cualquier proceso de trabajo dé como resultado un producto útil.

${ }^{3}$ El último trecho del informe del Banco Mundial citado dice: "But their fragile and limited resources, their often poorly defined property rights, and their limited access to credit and insurance markets prevent them from investing as much as they should in environmental protection" (World Bank, 1992: 30, citado en Anand y Sen, 2000: 2038). 
También permiten una reflexión sobre la actividad, una conciencia de los mecanismos internos (tecnología), y una permanente corrección del proceso y de los instrumentos utilizados para mejorar el producto final. Todas esas relaciones que el ser humano establece con el ambiente externo son relaciones técnicas, sea dicha naturaleza externa un medio biótico, ecosistemas con seres vivos, sea un medio abiótico, o sea una combinación de ambos. Además y simultáneamente a ese tipo de relaciones, los seres humanos establecen relaciones sociales de producción, por el simple hecho de que los medios con los cuales trabajan -sean éstos instrumentos, maquinaria, insumos o los propios espacios físicos en que se realizan las actividades- están distribuidos según reglas de propiedad y/o apropiación antes de ser realizada la actividad, y condicionan el reparto del producto y también el propio ritmo y tipo de técnica por utilizar. Una misma relación social de producción, como la relación capitalista, puede implicar relaciones técnicas de lo más variadas. La historia del capitalismo es elocuente en ello: pasó por las fases de uso de energía derivada del carbón vegetal, del carbón mineral, del petróleo, gas, energía eléctrica, energía nuclear, hidráulica, eólica, geotérmica, etcétera, sin por eso modificar las relaciones de propiedad y apropiación de los medios de producción: las relaciones sociales de producción continuaron siendo capitalistas.

Ahora podemos volver al triángulo (véase el esquema 1) para detectar algo que a primera vista no aparece explícito: la exclusión en la discusión sobre sustentabilidad de la posibilidad de cambios en las relaciones sociales de producción. Efectivamente, el extremo inferior derecho habla explícitamente de la sustentabilidad ecológica, o sea de la naturaleza externa al ser humano. Se trata, sin duda, de cambios en las relaciones técnicas. El extremo superior habla de sustentabilidad económica. No obstante, lo que se considera como sustentabilidad económica son cuestiones como la eficiencia, el crecimiento, o la retribución de los agentes del proceso con el valor aportado; en cualquier caso, cambios técnicos que no deben, ni pretenden, ni siquiera sugieren la posibilidad de cambios en las formas de propiedad de los medios de producción o de los recursos naturales. El ángulo inferior izquierdo habla de la sustentabilidad social. Sin embargo, la sustentabilidad social, a pesar de ser el ítem más confuso, lista una serie de elementos que tienden, todos ellos, a mejorar la calidad de vida, la democracia, o los derechos humanos, sin por eso tocar las relaciones de propiedad o apropiación de los recursos, y sin tocar las 
relaciones sociales de producción. Esto último queda explícito en el momento de analizar la evolución que el propio concepto de sustentabilidad social tuvo en las últimas décadas. De ello hablaremos en el siguiente apartado.

\section{Cambios en el concepto de sustentabilidad social en las últimas décadas}

Durante los últimos treinta años, la cuestión de la sustentabilidad social tuvo como eje central la pobreza y/o el incremento poblacional. Reducir la pobreza y limitar el crecimiento poblacional eran los objetivos de cualquier programa de sustentabilidad social. Obviamente, cuestiones como equidad social, calidad de vida, etcétera, estaban presentes; sin embargo, aquellos otros eran los temas centrales en el ámbito mundial. Como el incremento poblacional está directamente relacionado con la pobreza, ya que son los pobres quienes se reproducen a tasas elevadas, la pobreza era siempre la cuestión hegemónica. En términos teóricos pueden distinguirse dos fases en la concepción de la relación entre pobreza y degradación ambiental.

La primera va desde el surgimiento de la 'moderna' conciencia sobre la problemática ambiental en la década de los sesenta, hasta mediados de la década de los noventa. Durante esa fase, la visión hegemónica (ONU, Banco Mundial, etcétera) era conocida por la hipótesis de la 'espiral descendente' o del 'círculo vicioso'. Según esta hipótesis, los pobres son tanto agentes como víctimas de la degradación ambiental. Son agentes porque la falta de capital provoca que utilicen más intensivamente los recursos naturales y, consecuentemente, los depreden. En esa situación no tienen condiciones para "pensar en el futuro": deben pensar en la sobrevivencia cotidiana. Así, quedan progresivamente con menores recursos naturales para sobrevivir. Y son víctimas porque la escasez de dinero los obliga a migrar hacia áreas degradadas, más baratas. Por último, la falta de capital los obliga a compensar con más hijos que, a su vez, presionan sobre los mismos recursos naturales. Esta hipótesis del 'círculo vicioso' estuvo presente en la Conferencia de la ONU en Estocolmo en 1972, también en el informe Brundtland (1987), en el Reporte sobre Desarrollo y Medio Ambiente del Banco Mundial de 1992, y en el Informe sobre Pobreza y Medio Ambiente del PNud de 1995 (Angelsen, 1997). La alternativa para romper el círculo vicioso sería el desarrollo económico. 
La segunda fase comenzó a mediados y fines de la década de los noventa del siglo xx. La hipótesis del círculo vicioso fue revisada, mientras que la hipótesis del 'doble camino' (two-track approach) cobró hegemonía. Para ese cambio, el reconocimiento de varios aspectos fue fundamental. Entre ellos (Angelsen, 1997):

- Los bajos ingresos no siempre conducen a la degradación ambiental; tampoco los altos ingresos garantizan un equilibrio ambiental;

- La pobreza no debe ser considerada la causa principal de la degradación; hay que considerar las políticas gubernamentales, así como los grupos de poder y los sectores ricos;

- Tanto la pobreza como la degradación ambiental pueden tener una misma causa: falta de recursos o de derechos de propiedad sobre esos recursos;

- Ejemplos de sociedades agrícolas menos integradas al mercado muestran un mayor equilibrio ambiental: la degradación podría ser resultado de la integración mercantil.

Teniendo como punto de partida estas reflexiones críticas, la hipótesis del 'doble camino' muestra la necesidad de políticas públicas dirigidas explícitamente a combatir la pobreza en diferentes frentes simultáneamente: políticas de empleo, de vivienda, de educación, etcétera, deben ser implantadas. De alguna forma, este cambio de paradigma respecto de la relación entre pobreza y medio ambiente refleja una discusión más profunda, que tiene que ver con la diferencia entre garantizar a las futuras generaciones un mejor ambiente, o garantizar mejores generaciones. Mientras durante casi los últimos treinta años del siglo xx la discusión sobre el desarrollo sustentable puso el acento en la necesidad de legar a las futuras generaciones una naturaleza mejor -quedando la preocupación por el aumento de la calidad de vida en un mero medio para alcanzar aquella meta-, ya para finales del siglo xx la comunidad internacional comenzó a comprender que el objetivo debía ser el incremento de las capacidades humanas. El aumento de la calidad de vida debía ser el objetivo y no el puente o el medio para una naturaleza más saludable. El desarrollo humano, como aumento permanente de la cualidad humana en forma equitativa y, entonces, como objetivo propio, se coloca en primer lugar, y en la medida del desarrollo humano se alcanzaría una mejor relación con el ambiente externo (Anand y Sen, 2000).

Tanto en el caso de la hipótesis del 'círculo vicioso', como en la más reciente del 'doble camino', los posibles cambios no 
significan alteraciones de las relaciones sociales de producción. Esto puede ser visto más claramente al analizar uno de los elementos más radicales de la propuesta de sustentabilidad social: el de la participación social. Antes de abordar este tema, es preciso aclarar la completa incoherencia entre la propuesta del doble camino que supone una participación del sector público a través de políticas específicas para elevar la calidad de vida, y las medidas de política macro-económica impulsadas por esos mismos organismos internacionales, de carácter neoliberal, que en lugar de apoyar los gastos públicos, promueven las privatizaciones y la restricción del gasto fiscal. Es claro que las macro-políticas se imponen por sobre las primeras. La siguiente cita de un documento del Programa de las Naciones Unidas para el Desarrollo es clara en ese punto:

Macroeconomic reform can help a country become more competitive, but investing in the fastest-growing sectors can draw resources away from long-term investment in the resources of the poor... Countries may be tempted to overexploit natural resources to handle balance-ofpayment problems. And sometimes new and distant markets can encourage the depletion of a local resource to the detriment of the poor (UNDP, 1999).

De manera que todas las alternativas para combatir la pobreza van contra las macro-políticas impuestas por instituciones como el Fondo Monetario Internacional, o el Banco Mundial, las demandas de la Organización Internacional del Comercio, e inclusive las macro-recomendaciones de organismos como la FAO de la Organización de las Naciones Unidas. Elaboramos el cuadro 1 retomando, a partir del documento previamente citado de la UNDP, las causas de la pobreza consideradas en él; en la segunda columna relacionamos aquellas causas con las recomendaciones puntuales para combatir la pobreza del mismo informe; y en la tercera columna se muestran las ampliamente conocidas recomendaciones de macro-política económica (Fondo Monetario Internacional, Banco Mundial, Organización Mundial del Comercio). Es claro que la contradicción entre las dos últimas columnas es flagrante. 


\section{Cuadro 1}

Contradicción entre las propuestas y medidas sectoriales para combatir la pobreza y las macro-políticas económicas

\begin{tabular}{|l|l|l|}
\hline \multicolumn{1}{|c|}{ Causas de la pobreza } & \multicolumn{1}{|c|}{$\begin{array}{c}\text { Medidas de combate a la } \\
\text { pobreza según la UNDP }\end{array}$} & \multicolumn{1}{|c|}{$\begin{array}{c}\text { Macro-políticas } \\
\text { económicas } \\
\text { (FMI-BM-WTO) }\end{array}$} \\
\hline Menores oportunidades & $\begin{array}{l}\text { Obras públicas, obras de } \\
\text { infraestructura, tecnologías } \\
\text { apropiadas }\end{array}$ & $\begin{array}{l}\text { Reducción del gasto } \\
\text { público }\end{array}$ \\
\hline Desarrollo desigual & $\begin{array}{l}\text { Seguros, apoyo frente a } \\
\text { desastres, sistemas de transporte. } \\
\text { Áreas para vivienda cerca de los } \\
\text { locales de trabajo }\end{array}$ & Pago de la deuda externa \\
\hline $\begin{array}{l}\text { Distribución desigual de } \\
\text { recursos }\end{array}$ & $\begin{array}{l}\text { Reforma agraria, búsqueda de } \\
\text { mercados }\end{array}$ & Privatizaciones \\
\hline $\begin{array}{l}\text { Falta de derechos de } \\
\text { propiedad/uso }\end{array}$ & $\begin{array}{l}\text { Gobiernos democráticos y } \\
\text { garantía de derechos de } \\
\text { propiedad/uso }\end{array}$ & $\begin{array}{l}\text { Apertura comercial y } \\
\text { movimiento de capitales } \\
\text { sin restricciones }\end{array}$ \\
\hline Políticas erradas & $\begin{array}{l}\text { Co-manejo de recursos con los } \\
\text { pobres (participación). Co- } \\
\text { inversiones, } \\
\text { subsidios para defensa del medio } \\
\text { ambiente. Retirar subsidios a los } \\
\text { ricos y entregar a los pobres. } \\
\text { Políticas públicas de educación, } \\
\text { salud, etc. Aceptar soluciones } \\
\text { locales }\end{array}$ & $\begin{array}{l}\text { Desreglamentación del } \\
\text { trabajo y de las políticas } \\
\text { de bienestar social }\end{array}$ \\
\hline
\end{tabular}

Fuente: Elaboración propia con base en UNDP, 1999.

\section{Empoderamiento y gobernanza en la participación social}

Tal vez la participación social es la temática más interesante en la discusión sobre sustentabilidad social. La participación es un indicador de libertades democráticas, de equidad en las decisiones, y también un elemento decisivo en la potenciación de esfuerzos productivos. Ya desde la década de los ochenta del siglo Xx, el concepto de participación fue planteado por las agencias internacionales, organizaciones no gubernamentales (ONG), e instituciones internacionales, como un objetivo necesario de los programas de desarrollo y de sustentabilidad. Un informe de la FAO de 1981, por ejemplo, argumentaba:

participation by the people in the institutions and systems which govern their lives is a basic human right, and also essential for realignment of political power in favor of disadvantaged groups and for social and economic development (FERN, 2002: 9). 
Claro está que, en la práctica, el concepto de participación social fue mudando con el tiempo, desde una participación simplemente informativa hasta lo que hoy en día se conoce por la palabra empowerment o empoderamiento, que supone que los participantes de los proyectos de desarrollo sean de la naturaleza que fueren, discutan hasta las propias directrices estratégicas. ${ }^{4} \mathrm{El}$ cuadro 2 representa un esfuerzo de síntesis de la evolución del concepto de participación.

\section{Cuadro 2 \\ Tipología y evolución del concepto de participación}

\begin{tabular}{|l|l|}
\hline \multicolumn{1}{|c|}{ Tipología } & \multicolumn{1}{c|}{ Características } \\
\hline Participación pasiva & $\begin{array}{l}\text { Representantes no electos y sin poder. Reciben información } \\
\text { de los cambios sin poder de decisión. Contribuyen } \\
\text { eventualmente con recursos (materiales o con trabajo) sin } \\
\text { nuevo aprendizaje. }\end{array}$ \\
\hline $\begin{array}{l}\text { Participación } \\
\text { funcional }\end{array}$ & $\begin{array}{l}\text { Agencias externas que exigen participación para reducir } \\
\text { costos o con otros objetivos. Pueden eventualmente } \\
\text { participar en pequeñas decisiones, una vez que las } \\
\text { principales fueran definidas. }\end{array}$ \\
\hline $\begin{array}{l}\text { Participación } \\
\text { interactiva }\end{array}$ & $\begin{array}{l}\text { Participación conjunta en análisis y desarrollo de proyectos. } \\
\text { Fortalecimiento de instituciones locales. Metodologías } \\
\text { interdisciplinarias multi-propósito. En la medida en que los } \\
\text { grupos tienen control sobre las decisiones locales y } \\
\text { determinan cómo serán usados los recursos, tienen interés en } \\
\text { el mantenimiento de las estructuras o prácticas. }\end{array}$ \\
\hline $\begin{array}{l}\text { Movilización propia } \\
\text { self-movilization } \\
\text { empowerment }\end{array}$ & $\begin{array}{l}\text { Participación para cambiar sistemas con independencia de } \\
\text { las instituciones externas. Establecen contactos con } \\
\text { instituciones externas para asesorías técnicas y de recursos, } \\
\text { no obstante, conservan el control sobre cómo usar dichos } \\
\text { recursos. La auto-movilización se puede expandir si los } \\
\text { gobiernos y las OnG ofrecen soporte que lo permita. Esas } \\
\text { movilizaciones auto-iniciadas pueden o no cuestionar la } \\
\text { distribución de la riqueza y del poder. }\end{array}$ \\
\hline
\end{tabular}

Fuente: Adaptado de Pretty, 1995.

${ }^{4}$ Según Vogt y Murrell, el empoderamiento puede ser definido como sigue: "Empowerment is not merely an outcome but a process with its roots in the changing social, economic, and political structures of society. It is a way of being, a way of thinking -not merely a fad, tactic, or a quick fix. Empowerment, a personal, life long process that requires fundamental changes, and can be built only gradually. Each step must be solidly constructed, starting from the self; the individual must be self-confident, competent, and clear about the process of empowerment. Once the self is ready, the process can move on to relationships, groups, organizations, nations, and society as a whole" (Vogt y Murrell, 1990: 10). 
Es claro que hoy en día el último concepto de participación mostrado en el cuadro es el más divulgado, por lo menos en el discurso. No puede existir duda de que eso significa un avance civilizatorio significativo, frente a las antiguas versiones. No obstante, como explícitamente lo muestra Pretty -uno de los principales estudiosos de los procesos participativos en el ámbito rural-, ni el empoderamiento, ni la gobernanza significan por sí mismos alteraciones en las relaciones de propiedad y apropiación, o sea de las relaciones sociales de producción. ${ }^{5}$ Así, en poco o nada cambia el reparto de la riqueza social según las reglas de la competencia establecidas por el mercado, ni en sus consecuencias en lo que tiene que ver con la diferenciación social y la pobreza. Cierto es que una mayor conciencia social y organización social son elementos imprescindibles para cambios radicales; no obstante no conducen directa, ni necesariamente a ellos. La flagrante contradicción entre el discurso y la práctica queda evidente cuando movimientos sociales de alta participación, como el Movimiento de los Trabajadores sin Tierra del Brasil, dejan de encuadrarse en los criterios de empoderamiento y gobernanza y de ser sujetos de crédito por parte de la mayoría de las instituciones de crédito internacionales, a pesar de cumplir más claramente con los requisitos que esos conceptos encierran, como la libertad de acción, la autorresponsabilidad y la colaboración.

Cuando en el ámbito urbano las consignas más avanzadas de participación social reclaman la gobernanza, ahora en los ámbitos públicos también son restringidas a lo local, esfera que parece ganar prestigio como área de realización de la participación social, mientras que en los ámbitos mayores, o sea nacionales o regionales, las políticas neoliberales usan la gobernanza local para delegar funciones antes realizadas por los gobiernos centrales, y reducir así el presupuesto y déficit fiscales. Si, por un lado, se declara la gobernanza local como un ideal de expresión de la ciudadanía, por otro también se reclama la 'facilitación' (enablement) de mercado, o sea la posibilidad de que pequeñas y medianas empresas locales se integren directamente al mercado mundial, como si el mercado continuara siendo el mejor para asignar recursos.

Tampoco, obviamente, hay participación alguna en las decisiones importantes de las empresas capitalistas, que al final de

5 “....este concepto está dirigido a captar situaciones en que está en juego la coordinación entre actores independientes, de forma a lidiar con las cuestiones de la acción colectiva y de la cooperación" (Guimarães y Martin, 2001). 
cuentas son las que mueven toda la economía. Nuevamente algunos visionarios de la 'humanización' del capitalismo ya reclaman la necesidad de 'abrir' el funcionamiento de las corporaciones capitalistas a todo el público ("free markets cannot work behind closed doors"), como quien escribe:

Many of the issues I have just discussed [participation/governance] are relevant not only to governments, but also to the governance of corporations. Corporations are public institutions: they collect funds from the 'public' and invest them in productive assets. Workers too are stakeholders in corporations; given imperfections in labor mobility, a worker who is mistreated or fired cannot costlessly turn to other options (Stiglitz, 1999:4).

No obstante, la participación de los trabajadores en las corporaciones nunca podrá cambiar por causa de la participación interna o los procesos de empoderamiento, ya que las leyes capitalistas del reparto de la riqueza son establecidas como resultado de la competencia (o sea, de la predistribución privada de los medios de producción) y no de la suma de acciones individuales.

La gran cuestión en torno de la sustentabilidad social continúa siendo el hecho de que tal concepto se restringe a cambios técnicos, que pueden mejorar significativamente el nivel de calidad de vida en la esfera local, o para determinados sectores, pero, como escriben Middleton y O'Keefe, dos expertos en proyectos de desarrollo, no hay cómo atacar la justicia social sin afectar las relaciones de propiedad. En su libro Redefining Sustainable Development (2001) estos autores explican una contradicción clave: mientras los problemas de pobreza, justicia social y sustentabilidad social son, en términos generales, resultados sociales de una forma de reparto de los recursos y de los medios de producción, los discursos más avanzados del desarrollo sustentable, o las prácticas de las oNG de mayor compromiso con esos discursos continúan trabajando desde el individuo. Así, sucede que el mercado lanza, sistemáticamente y en forma creciente, trabajadores al desempleo, aumentando la pobreza, mientras que las propuestas de solución pretenden mitigar los resultados sin atacar las causas. El peso de las relaciones sociales de producción capitalistas restringe las posibilidades de que cambios en las relaciones técnicas alcancen la sustentabilidad social.

\section{Conclusiones}

En este breve artículo se mostró que el concepto de desarrollo sustentable incluye tres dimensiones básicas: la sustentabilidad 
ecológica, la sustentabilidad económica y la sustentabilidad social. De las tres, la de mayor controversia es la sustentabilidad social, ya que ha sido definida con base en conceptos no siempre claros. A pesar de eso, en los últimos años el concepto de sustentabilidad social evolucionó para resaltar la importancia de la participación social y del aumento de las potencialidades y cualidades de las personas en la construcción de un futuro más justo.

El propósito de este artículo fue mostrar que aun cuando han habido importantes avances, tanto en el ámbito teórico como en el de su instrumentación práctica, el desarrollo sustentable continúa básicamente anclado a un desempeño técnico, dentro de las reglas del juego del sistema de mercado capitalista, sin alcanzar ni cuestionar las relaciones de propiedad y apropiación capitalista, que generan pobreza, diferenciación social e injusticia.

En ningún momento pretendimos cuestionar la importancia de los procesos de desarrollo sustentable que impulsan una sustentabilidad social basada en los más modernos criterios de empoderamiento y gobernanza. Esos mecanismos y las agencias que los promueven pueden conducir a mejorías locales, de alto impacto en la población. Son también esenciales a los efectos de los derechos humanos, así como para la solución de problemas localizados de hambre o enfermedades específicas. No obstante, actúan sobre las consecuencias de un proceso de diferenciación social y de injusticia social, que es producto de las relaciones de mercado capitalistas. Por eso, por no afectar las propias relaciones sociales de producción que generan las desigualdades, su actividad tiene un enfoque técnico y además límites estructurales.

\section{Bibliografía}

Anand, Sudhir y Amartya Sen (2000), "Human Development and Economic Sustainability", World Development, vol. 28, núm. 12, Elsevier Science Ltd., Pergamon, Great Britain, pp. 2029-2049.

Angelsen, Arild (1997), "The poverty-environment thesis: was Brundtland wrong?", Forum for Development Studies, núm. 1, pp. 135-154.

Caldwell y L. Hon (1984), "Political aspects of ecologically sustainable development", Environmental Conservation, vol. 11, núm. 4, pp. 299-308. 
Daly, Herman, (1989), "Introducción a la economía en estado estacionario", en H. Daly (comp.), Economía, ecología, ética, Fondo de Cultura Económica, México.

FERN (2002), “Forests of Fear”, documento web: <www.fern.org/ Library/Reports/humanrights.pdf $>(05 / 02 / 2002)$.

Foladori, Guillermo y Humberto Tommasino (2000), "El concepto de desarrollo sustentable 30 años después", Cadernos de Desarrollo e Meio Ambiente, núm. 4, UfPr, Curitiba, Paraná, pp. 41-56.

Guimarães, Nadya Araújo y Scott Martin (Org.) (2001), Competitividade e Desenvolvimento. Atores e Instituições Locais, Editora SENAC, São Paulo.

Lélé, S. M. (1991), “Sustainable Development: a critical review”, World Development 19 (6), Pergamon Press, Great Britain, jun, pp. 607-621.

Middleton, Neil y Phil O'Keefe (2001), Redefining Sustainable Development, Pluto Press, London.

Pearce, D. y R. Turner (1995), Economía de los recursos naturales y del medio ambiente, Celeste Ediciones, Madrid.

Pimentel, D.; L. Westra y R. Noss (2000), Ecological Integrity. Integrating Environment, Conservation, and Health, Island Press, Washington, D.C.

Pretty, J. (1995), "Participatory learning for sustainable agriculture", World Development, vol. 23, núm. 8, pp. 12471263.

Rees, William y Mathis Wackernagel (1999), "Monetary analysis: turning a blind eye on sustainability", Ecological Economics 29, pp. 47-52.

Sachs, Ignacy (1994), "Estratégias de transição para o século XxI”, Cadernos de Desarrollo e Meio Ambiente, núm. 1, UfPR, Curitiba, pp. 47-63. 
Stiglitz, Joseph (1999), "Participation and Development. Perspectives from the Comprehensive Development Paradigm", Remarks at the International Conference on Democracy, Market Economy and Development, February 27, The World Bank Group, Seul.

UndP (United Nations Development Program) (1999), “Attacking Poverty while improving the Environment: towards winwin policy options”, Poverty \& Environment Initiative, UNDP.

Vogt, J.F. y K.L. Murrell (1990), Empowerment in Organizations, University Associates Inc., San Diego, California.

WCED (World Commision on Environment and Development) (1987), Our Common Future, Oxford University Press, Oxford.

WRI (World Resources Institute) (2002), Sustainability Simplified, documento web <www.wri.org $>$.

Enviado: 12 de mayo de 2002 Aceptado: 2 de junio de 2002 